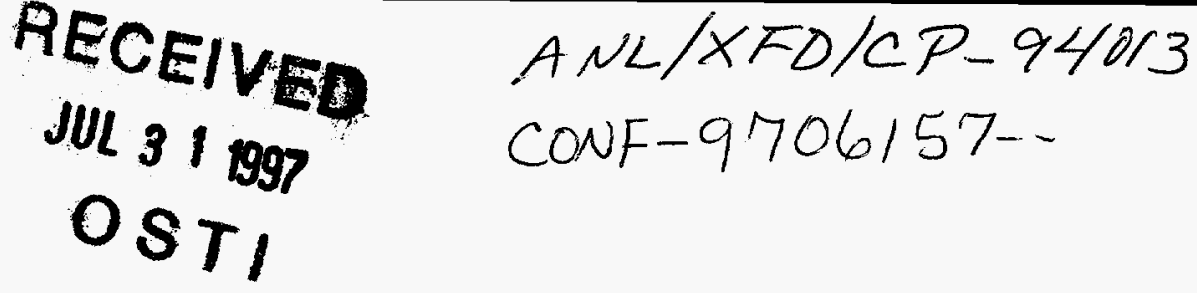

\title{
Progress of the APS High Heat Load X-ray Beam Position Monitor Development
}

\author{
Deming Shu, Juan Barraza, Hai Ding, Tuncer M. Kuzay, and Mohan Ramanathan
}

\author{
Experimental Facilities Division \\ Advanced Photon Source \\ Argonne National Laboratory \\ Argonne, Illinois 60439, U. S. A.
}

\begin{abstract}
The submitted manuscript has been authored by a contractor of the U.S. Government under contract No. W-31-109-ENG-38. Accordingly, the U.S. Government retains a nonexclusive, royalty-free license to publish or reproduce the published form of this contribution, or allow others to do so, for U.S. Government purposes.
\end{abstract}

\footnotetext{
Abstract: Several novel design developments have been established for the Advanced Photon Source (APS) insertion device (ID) X-ray beam position monitor (XBPM) to improve its performance:

- optimized geometric configuration of the monitor's sensory blades;

-- smart XBPM system with an intelligent digital signal processor, which provides a self-learning and calibration function; and

- Transmitting XBPM with prefiltering in the commissioning windows for the front end.

In this write-up, we summarize the recent progress on the XBPM development for the APS ID front ends.
}

\section{INTRODUCTION}

A third-generation synchrotron radiation source, such as the 7-GeV Advanced Photon Source (APS), generates high brilliance and intense synchrotron radiation from its insertion devices (IDs). There are many challenging tasks in the design of the ID beamline instrumentation that relate to high heat load and high heat flux problems. One of such component is the X-ray beam position monitor (XBPM) for the ID front ends and beamlines. The design requirements for APS front-end Xray beam position monitors (XBPM) are such that they must withstand the high thermal load (up to $600 \mathrm{Watts} / \mathrm{mm}^{2}$ ) and be able to achieve submicron spatial resolution while maintaining their stability.

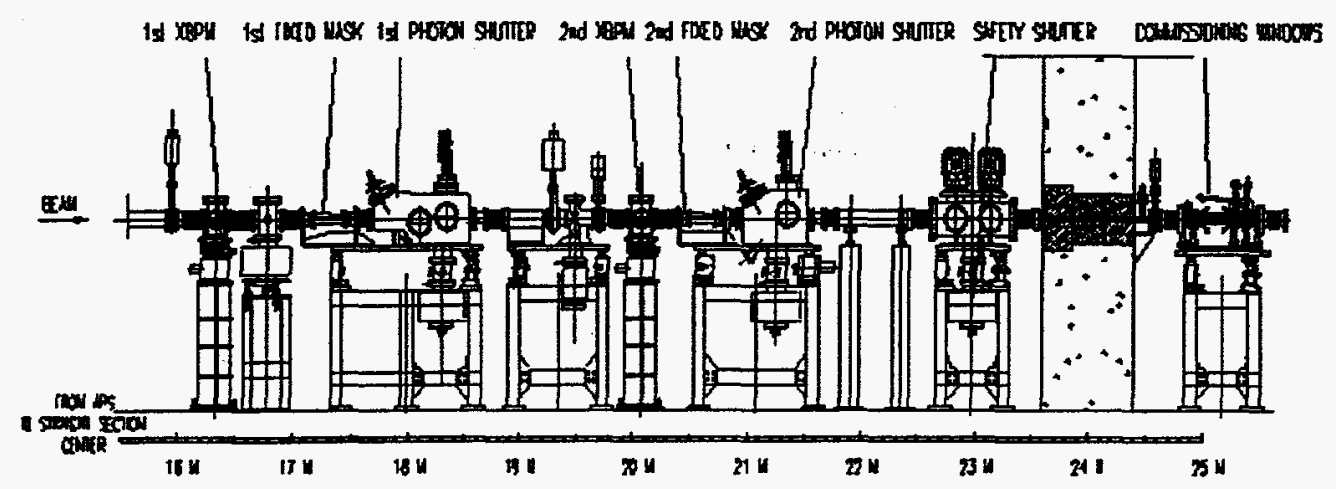

FIGURE 1. Schematic of the APS undulator beamline front end.

At the APS, each beamline front end has two XBPMs to monitor the X-ray beam position for both that vertical and horizontal directions. The XBPMs measure photoelectrons generated by the sensory blades and deduce the beam position by comparison of the relative signals from the blades. As shown in Fig. 1, both the first and second XBPM are located upstream of the user photon shutter (PS2) so that they are functional whether the user shutter is open or closed [1]. The major advantage of the XBPM is its high positioning sensitivity. Besides that, compared to the particle beam position monitors in the storage ring, the front end XBPMs have much higher sensitivity to the X-ray beam angular motion simply because they are located far away from the source.

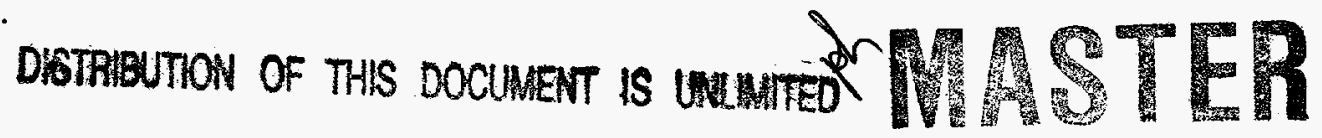




\section{DISCLAIMER}

This report was prepared as an account of work sponsored by an agency of the United States Government. Neither the United States Government nor any agency thereof, nor any of their employees, makes any warranty, express or implied, or assumes any legal liability or responsibility for the accuracy, completeness, or usefulness of any information, apparatus, product, or process disclosed, or represents that its use would not infringe privately owned rights. Reference herein to any specific commercial product, process, or service by trade name, trademark, manufacturer, or otherwise does not necessarily constitute or imply its endorsement, recommendation, or favoring by the United States Government or any agency thereof. The views and opinions of authors expressed herein do not necessarily state or reflect those of the United States Government or any agency thereof. 


\section{DISCLAMIER}

Portions of this docoment mory be illegible in electronic image products. Images are produced from the best available origiral docriment 
Additional design challenges for a conventional photoemmision type XBPM are the bending magnet contamination of the signal and its sensitivity to the ID gap variations. Work at other synchrotron radiation laboratories has shown that contamination signals caused by the bending magnet (BM) emitted radiation become a major problem [2]. Problems are exacerbated for the XBPM when the insertion devices (IDs) operate with different magnet gaps, because the percentage level of the contamination will be a variable.

There are several novel design developments established for the APS ID XBPM to improve its performance:

- Optimized geometric configuration of the monitor's sensory blades. function.

- Smart XBPM system with an intelligent digital signal processor, which provides a self-learning and calibration

--- Transmitting XBPM with prefiltering in the commissioning windows for the front end.

In this write-up, we summarize the recent progress on the XBPM development for the APS ID front ends in the mitigation of the problems explained in the foregoing.

\section{THE FRONT END XBPM STRUCTURAL DESIGN}

Since 1991, a number of the APS high heat load XBPM prototypes using CVD diamond as the blade material were tested successfully at CHESS and NSLS. Both analytical and experimental results proved that CVD diamond is a good choice for the APS high heat load XBPM blade material because of its superior thermophysical properties, such as: high thermal conductivity, low thermal expansion coefficient, good mechanical strength and stiffness under heat. Submicron position sensitivity was also demonstrated by the APS XBPM prototype using CVD diamond blades during CHESS and NSLS tests [3].

Fig. 2 shows the structure of the first XBPM (upstream) main assembly on the APS undulator beamline front end. In this

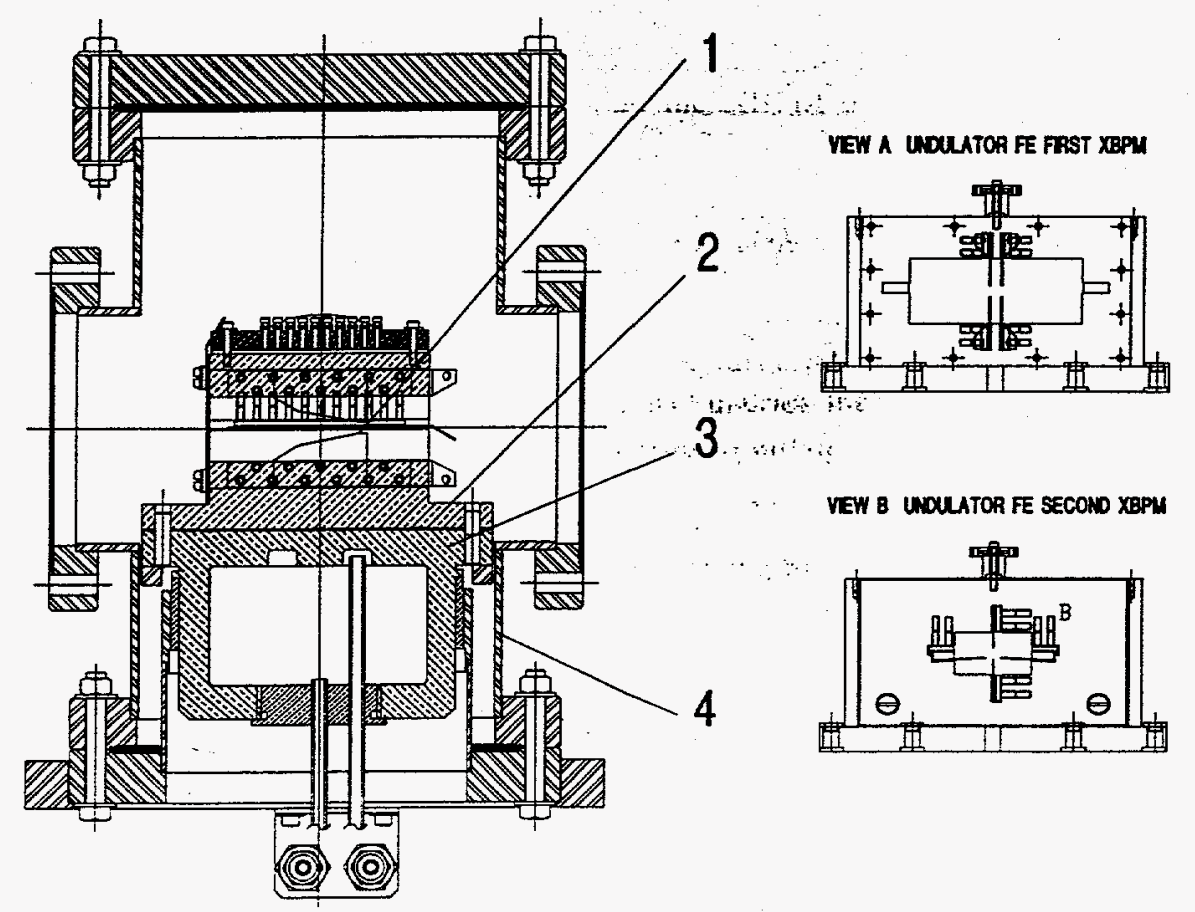

FIGURE 2. Structure of the XBPM main assembly for the APS undulator beamline front end, (1) CVD diamond blades, (2) monitor body, (3) water cooling base, (4) vacuum chamber. (view a for first XBPM monitor body, view b for second XBPM monitor body)

design, four $150-\mu \mathrm{m}$-thick CVD diamond blades (1) were coated with $1 \mu \mathrm{m}$ of gold. The blades are mounted vertically in pairs on the monitor body (2), which is made of oxygen-free copper (OFHC) and is cooled by a water cooling base (3) from the bottom. The vacuum chamber (4) and the cooling base are designed for ultrahigh-vacuum (UHV) condition. 
To eliminate the blade shadowing problems, the second XBPM (downstream) has a different blade placement configuration. As shown in Fig. 2b, the second XBPM has one pair of vertical blades, and one pair of "tilted" horizontal blades. This configuration reduces the signal contamination level from the BM-emitted radiation.

The XBPM monitor has the capability to apply a bias voltage. However, the test results show that a zero bias is acceptable and has the advantage of reducing the thermal resistance caused by the bias insulator. The geometrical configuration of the APS XBPM provides a low noise environment for photoelectron current output. The XBPM was sensitive enough to read out the photoemmission signal (about $0.6 \mathrm{nA}$ ) from a BM source while the APS storage ring had only a $24 \mu \mathrm{A}$ electron beam stored at the first APS X-ray test on March 26, 1995.

\section{STABILITY OF THE XBPM SUPPORTING STAGES}

As shown in Fig. 3, the XBPM main assembly (1) is supported by a precision supporting stage (2), which is mounted on top of a mounting post (3). The post is made of steel, filled on the inside with sand, and thermally insulated on the outside by ceramic cloth. This post design is very resilient to short-term temperature fluctuations.

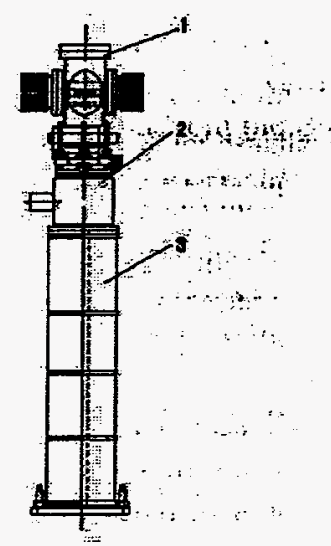

FIGURE 3. Front end XBPM with supporting stages, (1) XBPM main assembly, (2) precision supporting stages (3) mounting post.

The XBPM stage assembly consists of stepping-motor-driven vertical, horizontal, and rotational stages. Test measurements using a Laser Doppler Displacement Meter (LDDM) prove that the vertical stage attained a resolution of $<0.2$ $\mu \mathrm{m}$ with $1 \mu \mathrm{m}$ repeatability under a $200 \mathrm{lb}$ load [4]. Preliminary on-situ vibration tests show that the XBPM main assembly maintains less than $0.1 \mu \mathrm{m}$ rms vibration displacement level with the cóling water on.

\section{SMART XBPM SYSTEM (SBPM)}

The optimized geometric design for the blades helped reduce the BM contamination. For instance, on the first XBPM on the APS 1-ID front end, the BM contamination has been determined to be about $10 \%$ of the signal from the $2.4 \mathrm{~m}$ undulator A with a $15.8 \mathrm{~mm}$ magnet gap. However, the contamination level will be much higher when the undulator gap is opened more. The regular XBPM calibration process can only provide signal correction for one set of conditions. During normal operations, the insertion devices function at varying storage ring current, particle orbit and a variety of ID gaps. In addition, because of the expected imperfections in the ID magnetic field distribution, each ID and its location on the storage ring has its own "personality".

To offset the XBPM sensitivity to such operational variables, a newer XBPM system has been designed and a prototype built and tested for the APS. This new XBPM system has an intelligent signal processor, which provides a self-calibration function to serve as a noise and contamination signal rejecter to improve the system sensitivity and reliability [5].

The new APS XBPM system configuration is depicted in Fig. 4. It includes:

A. a pair of photo-electron emission-style beam position monitors using CVD diamond blades for undulator beamline front ends

B. a set of photo-electron current preamplifiers

C. a preamplifier auto-ranging controller and digitizer [6]

D. a digital signal processor (DSP) with EEPROM data base and ID source input interface for normalization [7]

E. a system controller with motor driver and encoder interface for XBPM calibration processes 
The new system, the so-called smart photon beam position monitor system (SBPM), has a built in EEPROM memory that is large enough to "remember" a complete calibration database covering all of the possible operating conditions. During the calibration mode, the monitor system controller initializes a series of automatic scan motions for the XBPM with different ID set-up information, and record them into the EEPROM database array. With the XBPM operating, the system corrects the normalized output according to the ID setup information and the calibration database. So that, with this novel system, the $\mathrm{XBPM}$ is always calibrated.

The heart of the smart system is a digital signal processor TMS320c40 from Texas Instruments Inc. [8], which is a floating-point processor designed specifically for digital parallel processing and real-time embedded applications. The key features of the TMS320c40 device, especially those to be used in the SBPM system, are the following: a high-performance DSP CPU with 40-ns instruction cycle times; a 40/32bit single-cycle floating-point/integer multiplier for high performance in computationally intensive algorithms; a six-channel DMA co-processor for concurrent VO and CPU operation; six communication ports for high-speed inter communication; 512byte on-chip program cache and 8 kbytes on-chip dualaccess/single-cycle RAM; two identical external data and address buses supporting a shared memory system with high data rate, etc..

In the operating mode, the DSP gets the XBPM signal data from the pre-amplifier/digitizer through one of the communication ports and groups them into an input buffer array.:Then the DSP calculates the data under the control of a signal normalization program, which is using the external EEPROM databaise for reference. After a step-by-step approaching process, the final beam position data (a pair for the beam positions at the first XBPM location and a pair for the beam angular displacement) is transmitted to a signal output buffer. There are two types of output data format available for users: 24-bit digital parallel output and 4-20 mA current loop for analog output. Both digital and analog output will keep the final beam position signal with a DC-50-Hz or a DC-300-Hz bandwidth (depending on the type of microprocessor to be used in the preamplifier controller).

\section{TRANSMTTTING XBPM FOR THE FRONT END COMMISSIONING WINDOWS (TBPM)}

During the beamline and front end commissioning activities, the final fine tuning of the storage ring and/or final adjustment of the front-end components is attempted. Based on the measurement data for the beam position in two locations

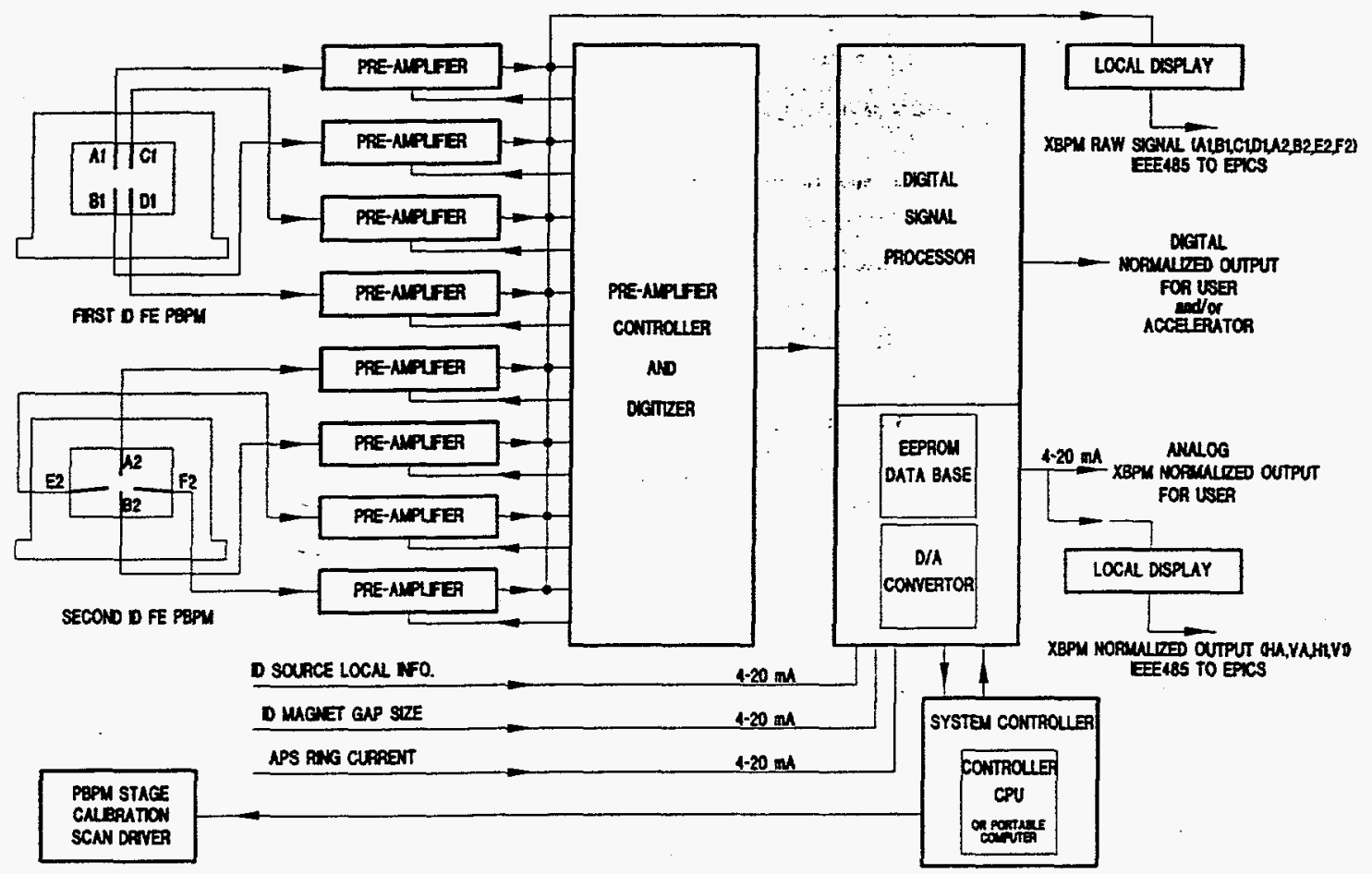

FIGURE 4. Schematic of the APS ID front end smart XBPM system. 
in an experimental station and in comparison with the calibration scan data from the front end X-ray BPMs, a new zero position is set after the synchrotron radiation beam commissioning.

A CVD diamond filter, which is a 25.4-mm diameter disk mounted on the downstream side of the fixed mask, is also designed as a transmitting $x$-ray beam position monitor (TBPM) for the APS commissioning window system [9]. The basic concept of the TBPM is to mount the monitor blade perpendicular to the synchrotron radiation beam and design the blade and its low-Z metal coating thickness in such a way that most of the X-ray beam is transmitted through the blade (just like filter or window). In this design, the $160-\mu \mathrm{m}$-thick CVD-diamond disk is coated with four electronically isolated aluminum quadrant patterns. The thickness of the aluminum coating is about $0.2 \mu \mathrm{m}$. The photoelectron emission signal is collected by a terminal interface disk, which is made from thin alumina and is coated with silver. This design concept provides the possibility of integrating the filter with TBPM functions. The beam position information from the TBPM in the commissioning window is very valuable to the front end commissioning and smart XBPM system initial calibration.

\section{DISCUSSION}

To date three smart XBPM systems have been installed on the APS ID front ends and they are operational. On-line preliminary tests began in August 1996. Rest of the $20 \mathrm{ID}$ front ends is expected to be furnished with SBPM system with DSP within a year. Based on the experience from the prototype operation, we will determine the time duration of the calibration period and optimize the database structure. Automatic calibration is necessary if the particle beam orbit changes frequently. If needed, the beam position at the neighboring bending magnet front end may also be used as another database reference input.

\section{ACKNOWLEDGEMENTS}

We acknowledge the help in the XBPM tests by Dr. Dean Haeffiner, and Messrs. Mark Keeffe, Michel Lehmuller, and Tim Cundiff. This work was supported by the U.S. Department of Energy, BES Materials Sciences, under Contract No. W31-109-Eng-38.

\section{REFERENCES}

1. D. Shu and T. M. Kuzay, Nucl. Instru. and Meth. A 347 (1994) 584.

2. T. Warwick et al. Rev. Sci. Instru. 66(2) Feb. 1995.

3. D. Shu, B. Rodricks, J. Barraza, T. Sanchez and T. M. Kuzay, Nucl. Instru. and Meth. A 319 (1992) 56.

4. T. Kuzay, ANL/APS TB-5, 1993.

5. D. Shu and T. M. Kuzay, Smart X-ray Beam Position Monitor System for the Advanced Photon Source, SRI95.

6. F. Meng, Unpublished M. Sc Thesis, IIT, May 1995.

7. X. Wu, Unpublished M. Sc Report, IIT, May 1996.

8. TMS320c4x User's Guide, Texas Instruments Inc. 1993.

9. D. Shu and T. M. Kuzay, Design of the Commissioning filter/Mask/Window Assembly for Undulator Beamline Front Ends at the Advanced Photon Source, SRI95. 